\title{
Sensitivity-Based Reliability Analysis of MEMS Acceleration Switch
}

\author{
Prashank Kansal ${ }^{1}$, Pramod Kasturi ${ }^{1}$, Nam H. Kim ${ }^{1} \&$ Seung-gyo Jang ${ }^{2}$ \\ ${ }^{1}$ Department of Mechanical and Aerospace Engineering, University of Florida, Gainesville, FL, USA \\ ${ }^{2}$ Agency for Defense Development, Daejeon, Republic of Korea \\ Correspondence: Nam H. Kim, Department of Mechanical and Aerospace Engineering, University of Florida, \\ Gainesville, FL, USA. Tel: 1-352-575-0665. E-mail: nkim@ufl.edu
}

Received: September 7, 2017

doi:10.5539/mas.v11n10p123
Accepted: September 18, 2017 Online Published: September 29, 2017

URL: https://doi.org/10.5539/mas.v11n10p123

\begin{abstract}
MEMS acceleration switches have been used in many engineering applications. In this paper, the reliability of MEMS switch is evaluated under various uncertainties from materials and manufacturing process. First, the performance of MEMS switch is modeled using 1D mass-spring-damper-contact system. Different from conventional lumped element methods, the model includes the effect of geometric parameters as well as contact conditions. The parameters of 1D models are calibrated using a high-fidelity finite element model. For reliability assessment, four different methods are used in order to compare computational cost as well as accuracy in predicting reliability. It turned out that sensitivity-based reliability method has several benefits, such as computational time and identifying important parameters that contribute significantly to reliability. The sensitivity analysis showed that the cross-sectional height and the length of folded-beam contribute most significantly to the reliability of switch-on condition. After changing the length of the beam, the probability of failure was improved from 0.168 to 0.0003 .
\end{abstract}

Keywords: MEMS acceleration switch, tolerances, uncertainty, reliability, parametric model

\section{Introduction}

The concept of micro-machined threshold accelerometer sensor was introduced back in 1972 (Frobenius, Zeitman, White, O'Sullivan \& Hamel, 1972), which was farfetched but well accepted. Since then, the MEMS technology has seen significant advancements in the past decades. The device proved to be cost-effective, efficient, robust and highly sensitive. It has been applied in various applications like seat belt tensioners, micro relays, airbag deployment (Selvakumar, Yazdi \& Najafi, K., 2000), transportation systems, arming and firing systems and medical industry (Zhou, Tu, Wong, Gong, Lian, He \& Ye, 2014). The basic concept of the device is to complete the circuit and activates as soon as the threshold acceleration or satisfying conditions are applied. However, the fabrication and reliability of the switch have posed some complications and have been a research topic over years.

Several papers have been published for designing and manufacturing MEMS acceleration switches. A silicon micro-machined threshold accelerometer is introduced by Loke et al. (1991) with silicon dioxide beams and fabricated on the surface of silicon wafer. The device was designed to detect high levels of acceleration. A new fabrication technology is described by Michaelis et al. (2000) and Tonnesen et al. (1997), called electroplating technology. It enabled the size of the switch to be reduced by a substantial amount, giving it a clear edge over conventional switches in automotive safety systems and transportation shock monitoring systems. Recently, a variety of methods, such as carbon nanotube contact pads (Lee, Song, Jung, Choi, Eun \& Kim, 2011; Lee, Eun, Jung, Choi \& Kim, 2011), squeeze film effect (Matsunaga \& Esashi, 2002) and movable contact pads (Cai, Ding, Yang, Su, Zhou \& Wang, 2008), have been adopted in acceleration switches to increase the contact time or 'switchON' time; thus providing stability, reliability and easy detection of threshold acceleration. These characteristics are crucial in many commercial applications like airbag restraint systems in transportation and fall detection in the medical industry. However, the sensitivity of the switch with respect to changes in material properties and manufacturing parameters is important for reliability of the switch, which is difficult to find in the literature.

MEMS devices come with a relatively large amount of manufacturing tolerances, which has a significant impact on the performance and function of the microsystem. The manufacturing tolerances can be classified into three 
categories, namely dimensional, geometric and alignment tolerances. Due to the small size of the microsystem, it is very hard to measure proper dimensional tolerances as they are of the order of micrometers, so proper research on this subject is desirable (Hsu, 2003). Geometric and alignment tolerances have a significant impact on assembly and packaging. The purpose of this paper is to evaluate the effect of these tolerances on the performance of the switch and perform reliability analysis to calculate the probability of failure.

In this paper, we will closely follow the horizontal acceleration switch design shown by Massad et al. (2005) in which the plate is suspended by four folded beams acting as springs and the base acceleration causes the plate to move forward and activate the switch. The length of the beam (910 970 microns) is very large compared to the height (4 8 microns), resulting in orders of magnitude difference in flexural rigidity between horizontal and vertical directions. In addition, due to the manufacturing process, it has a good control on manufacturing tolerances out of plane but large tolerances in plane. Random tolerances can affect the performance of switch in many ways. Therefore, reliability analysis of switch is conducted in this paper to quantify the effect of uncertainties in model parameters like modulus of elasticity, beam geometry, mass and identify critical design parameters.

The organization of the paper is as follows. In Section 2, a simple 1D MEMS switch model is presented whose parameters are calibrated based on finite element analysis. Section 3 discusses the sources of uncertainty in MEMS switch and reliability analysis using Monte Carlo simulation, Sensitivity-based Linear Approximate model, Firstorder Second Moment method, First-order Reliability method and Kriging surrogate model. Section 4 describes the improved design of MEMS switch based on sensitivity information. Lastly, Section 5 and 6 presents conclusions and acknowledgement.

\section{Performance Modeling}

\subsection{MEMS Switch Design and Finite Element Model}

The MEMS acceleration switch shown in Fig. 1 is designed such that when an acceleration in the range of $6 \mathrm{~g} \sim$ $10 \mathrm{~g}$ is applied, the gap between two electrodes is closed, by which the circuit is completed. The four folded beams are used to provide necessary stiffness for closing the electrodes. The performance of switch depends on the mass of moving part, the stiffness of folded-beam springs and the gap between two electrodes. There are a total of 36 comb structures at the both ends of moving plate, which can provide the effect of damping to the moving part. These comb structure can also protect the switch from accidental switch-on due to sudden impact or vibration. The moving plate has $17 \times 38$ etch holes (not shown in the figure) for the purpose of manufacturing.

Most parts of the MEMS switch are made of Silicon, except for electrodes, which is made of three layers of metal parts $(\mathrm{Ti} / \mathrm{Au} / \mathrm{Ni})$. The metal parts are used in order to reduce contact resistance, and thus, heat generation. In addition, the metal parts can prevent sticking each other, as well as enhance durability under repeated contacts.

A finite element (FE) model of MEMS switch is designed in Abaqus to calculate natural frequencies and static stiffness. The intended purpose is to calibrate and validate a single DOF model, which represents the first-order behavior of the switch, but includes model parameters so that reliability analysis can be performed in Section 4 . Therefore, an intermediate approach is undertaken in which we create an FE model with detailed geometry, from which a single DOF model can be designed.

For the purpose of FE modeling, the MEMS switch has been simplified by assuming the whole structure as a moving mass plate with four support beams, thirty-six brush beams and no etch holes. The support beam has a rectangular cross-section with z-directional thickness $b=60 \sim 80 \mu \mathrm{m}$ and y-directional height $h=4 \sim 8 \mu \mathrm{m}$ with clamped boundary conditions applied at four corners. All beams were modeled using quadratic beam elements. The moving plate is modeled using four-node quadrilateral elements. Since the etch holes are not playing any structural role, they are ignored in FE modeling. However, the density of Silicon is reduced in order to compensate for the missing etch holes. The effective area and density are computed by subtracting the area of holes from the area of the plate. For the electrodes, instead of modeling three layers of different material, a single layer is modeled with the average material property as shown in Table 1.

Table 1. Material properties of MEMS switch

\begin{tabular}{cccccc}
\hline \multirow{2}{*}{ Property } & \multicolumn{4}{c}{ Material } & Average \\
\cline { 2 - 5 } & Silicon & Titanium & Gold & Nickel & (Ti, Au, Ni) \\
\cline { 1 - 4 } Young's Modulus & 169 & 116 & 79 & 200 & 131.67 \\
$\begin{array}{c}(\mathbf{G P a}) \\
\text { Density }\left(\mathbf{g} / \mathbf{c m}^{\mathbf{3}}\right)\end{array}$ & 2.329 & 4.506 & 19.3 & 8.908 & 10.904 \\
\hline
\end{tabular}




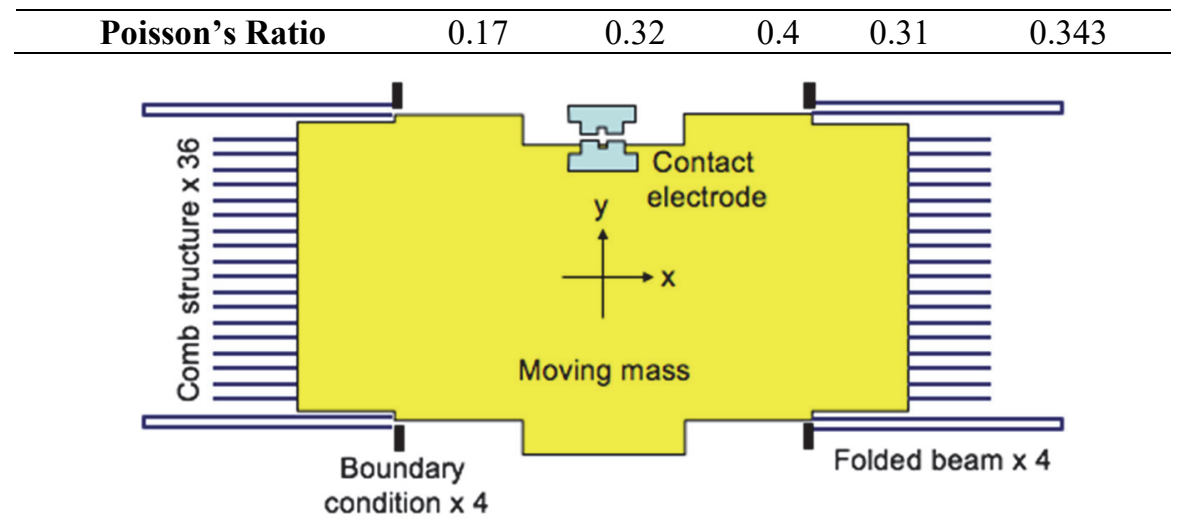

Figure 1. Acceleration MEMS switch model

\subsection{Modal Analysis}

The purpose of modal analysis is to show that the natural frequency of switching mode is much lower than other modes, and therefore, it can dominate the switch's dynamic response. The natural vibration frequencies and mode shapes are calculated in Abaqus. These two properties largely determine the dynamic motion of switch and help to validate the use of $1 \mathrm{D}$ mass-spring-damper-contact model. We checked the modes for 12 eigenvalues as they are enough to determine the motion of switch. Out of 12 modes, only four modes are related to the global deformation of the switch as they describe the motion of moving mass, and all others are local vibration modes of brush beams. Table 2 shows the natural frequencies and mode shapes of these four modes. The lowest frequency obtained is $687 \mathrm{~Hz}$, where four folded beams are in the bending mode in the same direction to the switching mode (see Fig. 2). The lowest natural frequency is about six times lower than the second lowest frequency. Therefore, the switch's motion will be dominated by switching directional translation, and thus, 1D mass-spring-damper model will be accurate to capture this motion.

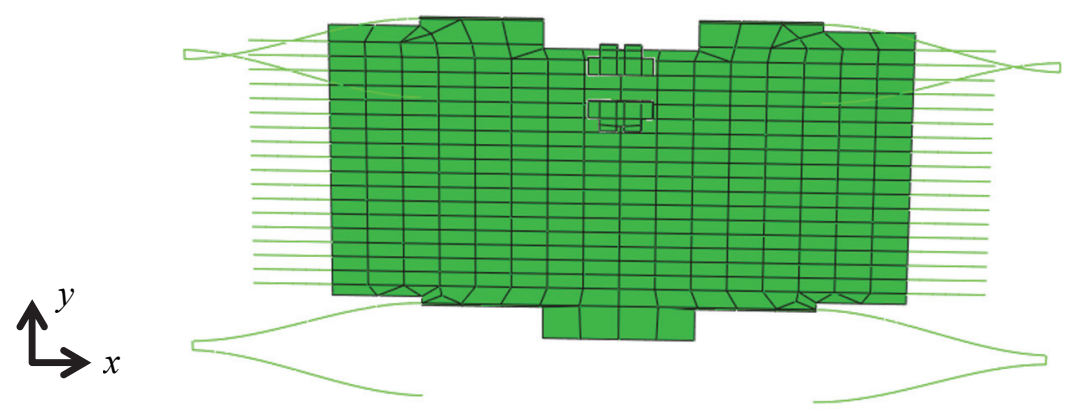

Figure 2. The first mode shape of switching direction

Table 2. Natural frequencies and deformation pattern corresponding to global deformation modes

\begin{tabular}{ccc}
\hline Mode & Natural Frequency & Description \\
\hline $\mathbf{1}$ & $687 \mathrm{~Hz}$ & Y-translation \\
$\mathbf{2}$ & $4296 \mathrm{~Hz}$ & Z-translation \\
$\mathbf{3}$ & $6973 \mathrm{~Hz}$ & X-rotation \\
$\mathbf{4}$ & $7549 \mathrm{~Hz}$ & Y-rotation \\
\hline
\end{tabular}

\subsection{Stiffness Analysis}

The purpose of stiffness analysis is to calculate an equivalent stiffness when the switch is modelled as 1D massspring-damper-contact system. The motion of switch would be dominated by the lowest stiffness. The stiffness is calculated by applying a specific load or moment in each coordinate direction and calculating the deflection or rotation. The stiffness in that direction can be obtained by dividing force applied (moment) by deflection (rotation) in the same direction. Table 3 gives a detailed information about the load applied and deflections obtained in 
different directions. For a constant force, stiffness is inversely proportional to the deflection. As can be clearly seen from Table 3, the stiffness in Y-direction (switching direction) is about 40 times smaller than the stiffness in Z-direction and 17 times smaller than stiffness in X-direction, making it the switching direction. This is due to the fact that deflection in Y-direction is much more as compared to other directions. It implies that the structure is suitable to approximate as a single DOF spring-mass-damping-contact system.

Table 3. Static stiffness calculated by applying loads/moments

\begin{tabular}{llll}
\hline Direction & $\begin{array}{l}\text { Load }\left[\mathbf{k g} \boldsymbol{\mu} \mathbf{m} / \mathbf{s}^{\mathbf{2}}\right] \\
\text { Moment }\left[\mathbf{k g} \boldsymbol{\mu} \mathbf{m}^{2} / \mathbf{s}^{2}\right]\end{array}$ & $\begin{array}{l}\text { Deflection }[\boldsymbol{\mu m}] \\
\text { Rotation }[\mathbf{r a d}]\end{array}$ & $\begin{array}{l}\text { Stiffness }[\mathbf{N} / \mathbf{m}] \\
\text { Rotational Stiffness }\left[\mathbf{k g} \boldsymbol{\mu \mathbf { m } ^ { 2 }} / \mathbf{s}^{2}\right]\end{array}$ \\
\hline X-translation & 100 & 1.055 & 94.79 \\
Y-translation & 100 & 15.74 & $\mathbf{6 . 3 5}$ \\
Z-translation & 100 & 0.4484 & 223.02 \\
X-rotation & 100 & $1.751 \mathrm{E}-6$ & $5.71 \mathrm{E} 7$ \\
Y-rotation & 100 & $8.759 \mathrm{E}-7$ & $1.14 \mathrm{E} 8$ \\
Z-rotation & 100 & $3.658 \mathrm{E}-6$ & $2.73 \mathrm{E} 6$ \\
\hline
\end{tabular}

\subsection{Analytical Model and Calibration of Folded-beam}

The stiffness obtained in the switching direction is $\mathrm{K}_{\mathrm{FEA}}=6.35 \mathrm{~N} / \mathrm{m}$ in FE analysis when a load of $100 \mu \mathrm{N}$ is applied, but this stiffness is for a specific geometry of beam cross section. In order to avoid running FE analysis repeatedly for different geometries, we came up with an analytical model of the four folded beams to express deflection and stiffness in terms of material and geometric properties. By assuming that the folded beam is a serial connection of two beams, the half span of the folded beam is modeled using an Euler beam with one end fixed and the other end attached to roller boundary condition and applied force to the tip in the y-direction (Figure 3 ).

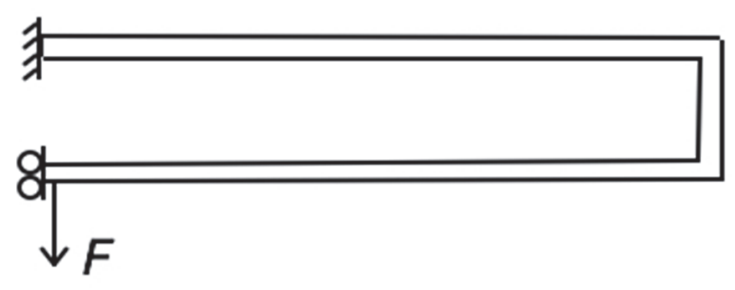

(a)

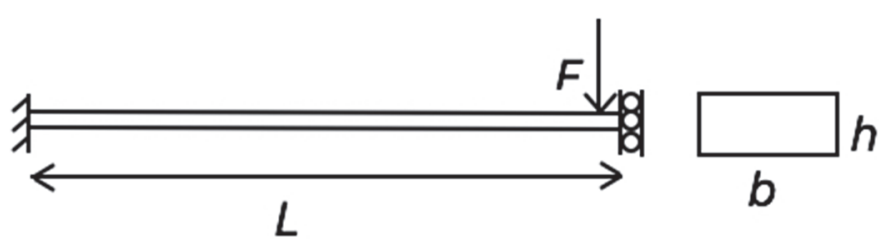

(b)

Figure 3. Approximation of a folded beam. (a) Folded beam, (b) Half model of folded beam with cross section

The deflection of a single beam (Fig. 3(b)) can be analytically obtained using:

$$
v(x)=\frac{F}{12 E I}\left(2 x^{3}-3 L x^{2}\right)
$$

By dividing the applied force with the deflection at $x=L$, the following beam stiffness can be obtained:

$$
K_{\text {Analytical }}=\frac{F}{v(L)}=\frac{12 E I}{L^{3}}
$$

In the above equations, $F$ is the applied load, $E$ the Young's modulus, $L$ the length of beam, and $I$ the moment of inertia. The advantage of using Eq. (2) is that when the geometry of beam changes, it is still possible to calculate the stiffness analytically.

For the given dimensions, the stiffness obtained using Eq. (2) is $3.35 \mathrm{~N} / \mathrm{m}$ for a single beam. By assuming that the 
folded beam is equivalent to a series connection of two beams, the stiffness is halved (3.35/2). In addition, since the MEMS switch consists of four folded beams connected in parallel, the total stiffness of the switch would be four times the stiffness of a single beam. Therefore, the stiffness of the switch is $6.72 \mathrm{~N} / \mathrm{m}$ calculated analytically.

Due to the incomplete representation of folded beam configuration and boundary conditions, there is a difference between stiffness for analytical method $\left(\mathrm{K}_{\text {Analytical }}=6.72 \mathrm{~N} / \mathrm{m}\right)$ and that from finite element model $\left(\mathrm{K}_{\mathrm{FEA}}=6.35\right.$ $\mathrm{N} / \mathrm{m}$ ). The difference may come from the detailed geometry where two beams are connected as well as from the boundary conditions. We compensate for this discrepancy between the two values of stiffness by introducing a correction factor, which is defined as the ratio between $\mathrm{K}_{\mathrm{FEA}}$ and $\mathrm{K}_{\text {Analytical. }}$. For this example, the correction factor becomes 0.95 , which means that the stiffness from both models are close enough and hence they validate each other. The correction factor will be multiplied to the stiffness of 1D spring-mass-damper-contact system in order to match the model performance with that of finite element analysis.

\subsection{Performance Modeling}

The purpose of performance modeling is to approximate the single DOF spring-mass-damper-contact system on MATLAB using ode 45 solver and obtain the displacement-time plots, which is in agreement with the plots obtained through FE analysis for various accelerations. Based on modal and static analysis, the MEMS switch can be modeled as a single DOF spring-mass-damper-contact system, as shown in Figure 4 (Kim, 2004).

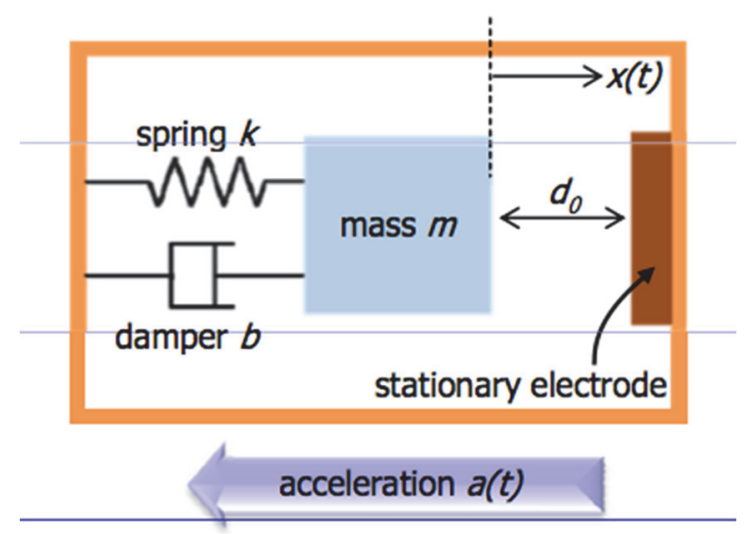

Figure 4. 1D dynamic model of acceleration switch

The governing differential equation of this system is given by

$$
m \frac{d^{2} x}{d x^{2}}+c \frac{d y}{d x}+k x=m a(t)+F_{c}(x)
$$

where, $m$ is the proof mass, $c$ the damping factor, $k$ the stiffness, $x$ the motion of mass, and $F_{c}$ the contact force. The stiffness of the spring is calculated using the procedure described in Section 2.4, while the damping factor $\mathrm{c}$ is calculated by comparing the behavior of the mass against experiment.

When the moving mass hits the stationary electrode, a contact force is generated which is modeled using Hertz model. A contact force model with hysteresis damping, discussed by Hamid and Parvis (Hamid \& Parviz, 1994), is used in this paper, as

$$
F_{c}=K_{c} \delta^{1.5}\left(1+\frac{3\left(1-e_{r}^{2}\right)}{4} \dot{\delta}\right)
$$

where, $K_{c}$ is the contact stiffness, $\delta$ the penetration defined as $\delta=<x-d_{0}>_{+}, \dot{\delta}$ the rate of penetration and $e_{r}$ the elastic coefficient of restitution. The angled bracket $\langle a\rangle_{+}$means that the value is zero when $a<0$, while the value itself when $a>0$. That is, the penetration is zero when the moving mass is separated from the electrode, while positive when the moving mass penetrates the electrode. The penalty-based contact formulation allows a small penetration and the contact force is proportional to the penetration.

During the steady-state response, the dynamic equation reduces to $k x=m a$, assuming that the contact does not occur. Therefore, the threshold or switching 'on' acceleration can be determined by 


$$
a_{t h}=\frac{k}{m} d_{0}
$$

where, $d_{0}$ is the initial gap between stationary and moving mass.

The industry requirements say that switch-on acceleration should lie within $6 \mathrm{~g} \sim 10 \mathrm{~g}$, failing to do so will result in the failure of the switch. Figure 5 shows the displacement time plot at $6.30 \mathrm{~g}$ and $8.84 \mathrm{~g}$. As shown, $6.3 \mathrm{~g}$ acceleration is just enough to turn on the switch and achieve maximum deflection but does not maintain a continuous contact and bounces back to $3.6 \mu \mathrm{m}$, and then reaches steady state at a deflection of $4.3 \mu \mathrm{m}$. On the other hand, with an acceleration of $8.8 \mathrm{~g}$, the switch reaches maximum deflection at $0.5 \mu \mathrm{sec}$, bounces back to $5.1 \mu \mathrm{m}$, then reaches steady state and maintain continuous contact. Therefore, the switch would be continuously on when $10 \mathrm{~g}$ of acceleration is applied. A comparison between steady state deflection obtained from FE analysis and performance model is shown in Table 4. The values obtained from FE Analysis and the performance model are in agreement, which shows that the performance model depicts the behavior of the switch appropriately. Therefore, the assumption of MEMS Switch as a 1D mass-spring-contact-damper system is valid.

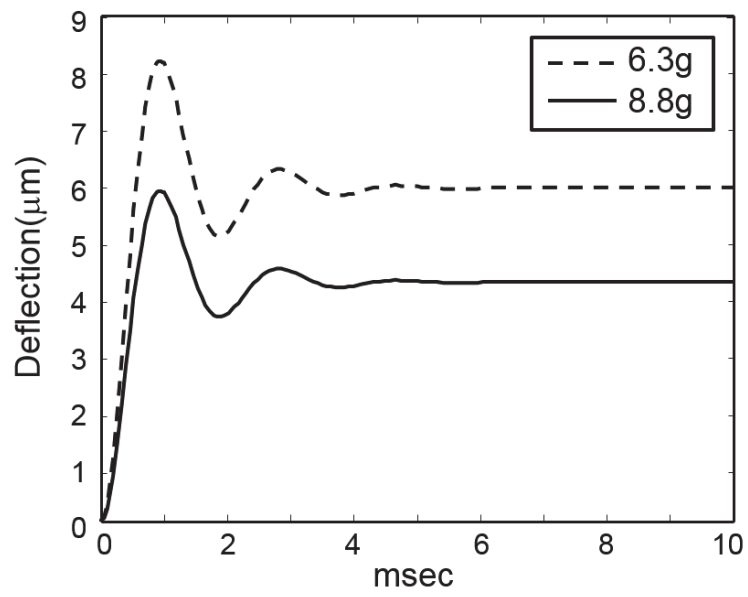

Figure 5. Time-deflection curves of switch for different accelerations

Table 4. Comparison between steady-state deflection obtained from the performance model and FE analysis

\begin{tabular}{ccc}
\hline \multirow{2}{*}{ Acceleration } & \multicolumn{2}{c}{ Steady State Deflection } \\
\cline { 2 - 3 } & Performance model & FE Analysis \\
\hline $6.3 \mathrm{~g}$ & $4.3 \mu \mathrm{m}$ & $4.3 \mu \mathrm{m}$ \\
$8.8 \mathrm{~g}$ & $6.0 \mu \mathrm{m}$ & $6.0 \mu \mathrm{m}$ \\
\hline
\end{tabular}

\section{Sources of Uncertainty and Reliability Analysis}

\subsection{Source of Uncertainty in MEMS Switch}

Industry demands MEMS devices to be reliable as they are often used for safety-related systems, but at the same time due to the micro size nature, it is very difficult or expensive to produce them without any variability in manufacturing. This variability can cause the performance of switch to fluctuate and result in failure. This paper aims at quantifying the probability of failure by relating the uncertainty in input parameters to that in output. In a MEMS switch, there are many sources of uncertainty, such as Young's modulus, Poisson's ratio, dimensions, the gap between electrodes and operating environment (Agarwal \& Aluru, 2009). For example, the change in support beam dimension can change the stiffness, and thus, the response of the switch. This is shown in Table 5 where a small change in beam cross-section leads to a substantial change in stiffness and 'switch-on' acceleration. Thus, it is important to quantify the effect of input parameters' uncertainty on the performance of switch in order to manufacture reliable MEMS switches.

Among various uncertain input parameters, three parameters related to the geometry of supporting beams, two material parameters and the gap between moving mass and stationary electrode are considered in this paper. Table 6 shows these uncertain parameters with their statistical distribution parameters. These uncertain parameters represent manufacturing tolerances and variability in material properties. In order to protect proprietary 
information, ranges of means are given in Table 6 .

Table 5. Change in stiffness and required acceleration for different supporting beam cross-section

\begin{tabular}{ccc}
\hline Cross-section & Lateral Stiffness & Switch-on Acceleration \\
\hline $6 \times 70 \mu \mathrm{m}^{2}$ & $6.35 \mathrm{~N} / \mathrm{m}$ & $7.46 \mathrm{~g}$ \\
$7 \mathrm{x} 70 \mu \mathrm{m}^{2}$ & $9.52 \mathrm{~N} / \mathrm{m}$ & $11.85 \mathrm{~g}$ \\
\hline
\end{tabular}

Table 6. Uncertainty in material properties and geometry

\begin{tabular}{lcc}
\hline Input Parameters & Mean Value & CoV \\
\hline Beam Length (L) & $910 \sim 970 \mu \mathrm{m}$ & 0.1 \\
Beam Width (b) & $60 \sim 80 \mu \mathrm{m}$ & 1 \\
Beam Height (h) & $4 \sim 8 \mu \mathrm{m}$ & 0.1 \\
Young's Modulus & $(160 \sim 180)^{*} 10^{3}\left(\mathrm{~kg} / \mu \mathrm{ms}^{2}\right)$ & $1 \%$ \\
(E) & $(4 \sim 5)^{*} 10^{-7} \mathrm{~kg}$ & $0.0589 * 10^{-7}$ \\
Mass (m) & $4 \sim 8 \mu \mathrm{m}$ & 0.1667 \\
Initial Gap (gap) & & \\
\hline
\end{tabular}

There are a variety of ways to perform reliability analysis and quantify uncertainty in output, but we will follow four approaches in this article. They are classified as: (1) Monte Carlo Simulation; (2) Sensitivity-based Linear Approximation and First-order Second Moment; (3) First-order Reliability Method; and (4) Surrogate-based Monte Carlo Simulation. Other methods include Importance Sampling, Second-order Reliability Method, etc. We chose these methods based on the popularity and ease of use.

\subsection{Reliability Analysis using Monte Carlo Simulation}

Monte Carlo Simulation is a conventional solution to measure the uncertainty in simulation outputs, but it is computationally expensive and time inefficient. Monte Carlo is a method, which predicts the outcomes of all possibilities by repeating the calculation hundreds or thousands of times according to the input probability distribution. It chooses an input sample set from the probability distribution and propagates it through the system model to obtain a sample of output. Monte Carlo Simulation does this numerous times and generates a comprehensive view of what the output distribution can be. We can then determine how many of them satisfy the performance requirement (reliability) and how many of them failed (probability of failure). Let the system performance function $G(\mathbf{x})$ is defined as

$$
G(\mathbf{x})=d(\mathbf{x})-\text { gap }
$$

where $d(\mathbf{x})$ is the y-directional displacement of moving mass, gap is the initial gap between electrodes, and $\mathbf{x}$ is the vector of input random variables. The vector of input variables $\mathbf{x}=\{L, b, h, E, m \text {, gap }\}^{\mathrm{T}}$ is random variables whose distribution parameters are given in Table 6 . The system is considered to be failed when $G(\mathbf{x})<0$ for an acceleration of $10 \mathrm{~g}$ or $G(\mathbf{x})>0$ for $6 \mathrm{~g}$ at $10 \mu \mathrm{sec}$. Both events are mutually exclusive so individual failure probabilities are added to obtain the system failure probability.

In MCS, $N$ samples are generated and the probability of failure $P_{F}$ is calculated. However, this process is time consuming and an expensive way to perform risk analysis, which is not suitable when many samples are involved. When a finite number of samples are used, Monte Carlo simulation results have sampling error; that is, the results are different every time when the process is repeated. Therefore, the concept of confidence intervals is used to estimate the error in Monte Carlo simulation results. Confidence interval describes the amount of uncertainty associated with the statistical prediction of reliability from samples. As the number of samples increases, the confidence interval will decrease.

In this paper, the steady-state deflection of the beam is calculated when a constant acceleration of $6 \mathrm{~g}$ and $10 \mathrm{~g}$ is applied. Input variables and the initial gap are normally distributed with the mean and the coefficient of variance as shown in Table 6 . The number of samples is determined for which the performance function $G(\mathbf{x})$ is greater than 0 and less than 0 with an acceleration of $6 \mathrm{~g}$ and $10 \mathrm{~g}$, respectively. The probability of failure is then computed using

$$
P_{F}=\frac{\text { number of failed samples }}{\text { total number of samples }}
$$


The system probability of failure is computed by adding both probabilities as they are mutually exclusive. 142 samples are failed out of 1000 samples, which means $P_{F}=0.142$. The confidence interval of the Monte Carlo simulations helps to provide an estimate of the true failure probability. Depending on the confidence level, the true probability of failure will lie within the interval for given number of samples. For example, a confidence level of 95\% means that the probability of true P_F being within the interval is $95 \%$. Confidence interval is calculated using Wald method which is the most common method (Dunnigan, 2008). It is given by the following formula:

$$
\text { Interval }=P_{F} \pm z_{\frac{\alpha}{2}} \sqrt{\frac{P_{F}\left(1-P_{F}\right)}{N}}
$$

where $N$ is the number of samples and $z_{\alpha / 2}$ is the margin of error, which depends on the confidence level. In the case of $95 \%$ confidence level, $z_{\alpha / 2}=1.96$. Using Eq. (8), the $95 \%$ confidence interval with 1000 samples becomes $[0.112,0.172]$. Although this method is fast, it may not be useful when the interval is large compared to the level of probability of failure. With 1000 samples, the interval is about $40 \%$ of the failure probability.

\subsection{Reliability Analysis Using Sensitivity-based Linear Approximation and First-Order Second Moment}

The purpose of this method is to find the probability of failure of MEMS switch using linear approximation and first-order second moment. It can be seen from Eqs. (2) and (5) that the steady-state deflection is a function of the modulus of elasticity, beam cross-section, beam length and moving mass. In this section, we will approximate the steady-state deflection as a linear function of these input variables using sensitivity (gradient) and the first-order Taylor series expansion. The performance of switch is determined by comparing the steady-state deflection of the moving mass with the initial gap between the stationary electrode and moving mass. The switch fails if the deflection is less than the initial gap for $10 \mathrm{~g}$ acceleration or deflection is larger than the initial gap for $6 \mathrm{~g}$ acceleration.

\subsubsection{Sensitivity and Finite Differences}

Sensitivity is defined as the rate at which the function changes with respect to the input variables, $\left(\partial G / \partial x_{i}\right)$, assuming that the function is differentiable and continuous. Sensitivity can be calculated using three approaches: the approximation, discrete and continuum approach (Choi \& Kim, 2004). We will use the approximation approach where the sensitivity is obtained using the finite difference method. The finite difference method is the easiest and most common method for evaluating sensitivity. It evaluates sensitivity by computing function value at different intervals of the input variable. If each variable $x_{i}$ is perturbed by a small step $\Delta x_{i}$, the sensitivity can be approximated by:

$$
\frac{\partial G}{\partial x_{i}} \approx \frac{G\left(x_{i}+\Delta x_{i}\right)-G\left(\Delta x_{i}\right)}{\Delta x_{i}}
$$

This is called forward difference method (FDM) as the perturbation is in the forward direction. If the variable is perturbed in both positive and negative directions, it is called central difference method (CDM) and is given by:

$$
\frac{\partial G}{\partial x_{i}} \approx \frac{G\left(x_{i}+\Delta x_{i}\right)-G\left(x_{i}-\Delta x_{i}\right)}{2 \Delta x_{i}}
$$

In general, it is known that $\mathrm{CDM}$ is more accurate than $\mathrm{CDM}$, but the latter requires a more number of function evaluations. There are some disadvantages of calculating the sensitivity using the finite difference method. For a nonlinear function, the accuracy of sensitivity in Eqs. (9) and (10) depends on the step size $\Delta x_{i}$; therefore, a small step size should be used. However, a too small step can cause an error due to numerical truncation. Therefore, it is important to choose an appropriate step size based on numerical experiments.

In this paper, $\mathrm{CDM}$ is used to calculate the sensitivity because the error in CDM is of the order of $\Delta x_{i}^{2}$, while in FDM it is of the order of $\Delta x_{i}$. Table 7 shows the sensitivity of the steady-state deflection with respect to all input variables. The CDM with a step size of $1 \%$ is used to calculate the sensitivity of deflection with respect to five random variables. Notice that the sensitivity with respect to Young's modulus, beam height and width is negative as they are inversely proportional to the deflection. Also the sensitivity with respect to beam height and length is large compared to others, which means they are critical design parameters of the MEMS switch.

Table 7. First-order sensitivity of steady-state deflection

\begin{tabular}{cc}
\hline Input variable & Sensitivity \\
\hline $\begin{array}{c}\text { Young's Modulus } \\
\text { (E) }\end{array}$ & -0.32 \\
\hline
\end{tabular}




\begin{tabular}{cc}
\hline Beam width (b) & -0.32 \\
Beam height (h) & -0.96 \\
Beam Length (L) & 0.95 \\
Proof mass (m) & 0.32 \\
\hline
\end{tabular}

\subsubsection{Linear Approximation}

Since the original nonlinear performance model is expensive to evaluate, it is linearized with respect to the nominal value of input variables, $\mathbf{x}_{0}=\left\{E_{0}, b_{0}, h_{0}, L_{0}, m_{0}, \text { gap }_{0}\right\}^{\mathrm{T}}$, using sensitivity information. Linearization not only provides a computational efficiency but also a convenient property in reliability analysis. When input random variables are normally distributed, their linear combination is also normally distributed. Therefore, the reliability of a linear performance function can be analytically calculated. Of course, the linear approximation can cause a large error for highly nonlinear function, but if the standard deviation of input random variables is small, the error can be small. Also, the linear approximation can induce a large error when interaction between different input variables is high.

The performance function is linearly approximated using Taylor's theorem and sensitivity information as

$$
\tilde{G}(\mathbf{x}) \approx G\left(\mathbf{x}_{0}\right)+\left\{\frac{\partial G\left(\mathbf{x}_{0}\right)}{\partial \mathbf{x}}\right\}^{\mathrm{T}}\left(\mathbf{x}-\mathbf{x}_{0}\right)
$$

Note that the performance function is already linear with respect to the gap. Note that if the input variables, $\mathbf{x}$, are normally distributed, the linearize performance function, $\widetilde{G}(\mathbf{x})$, is also normally distributed.

\subsubsection{First-Order Second Moment Method (FOSM)}

This method is also called as Mean Value First-Order Second-Moment method because of the fact that the function is linearized about the mean value of the random input variables. Equation (11) linearizes the performance function about the mean value of six normally distributed input random variables. The MEMS switch fails when $G(\mathbf{x})<0$ for $10 \mathrm{~g}$ and $G(\mathbf{x})>0$ for $6 \mathrm{~g}$, respectively. Then, the probability of failure is given by:

$$
P(G(\mathbf{x})<0)=\int_{G(\mathbf{x})<0} f_{\mathbf{X}}(\mathbf{x}) \mathrm{d} \mathbf{x}
$$

for acceleration of $10 \mathrm{~g}$, and

$$
P(G(\mathbf{x})>0)=\int_{G(\mathbf{x})>0} f_{\mathbf{x}}(\mathbf{x}) \mathrm{d} \mathbf{x}
$$

for acceleration of $6 \mathrm{~g}$. In Eqs. (12) and (13), $f_{\mathbf{X}}(\mathbf{x})$ is the joint probability density function (PDF) of $\mathbf{x}$. The reliability index $(\beta)$ is defined by the ratio of mean and standard deviation of performance function. Since the performance function is linearized, the standard deviation is calculated by the following equation:

$$
\sigma^{2}=\sum_{i=1}^{n}\left(\frac{\partial G\left(\mathbf{x}_{0}\right)}{\partial x_{i}}\right)^{2} \sigma_{x_{i}}^{2}
$$

where the input variables are assumed to be uncorrelated.

In the case of acceleration $10 \mathrm{~g}$, the mean and standard deviation turned out to be $0.374 \mu \mathrm{m}$ and $0.383 \mu \mathrm{m}$, respectively, which yields the reliability index $\beta$ of 0.975 . The probability of failure is calculated by taking the standard normal CDF of the negative reliability index. Thus, the probability of failure of MEMS switch for an acceleration of $10 \mathrm{~g}$ is 0.1648 . Similarly, the value of $\beta$ for $6 \mathrm{~g}$ is calculated to be 6.532 which gives the probability of failure as $3.288 \mathrm{E}-11$, which means that the probability of switch-on at $6 \mathrm{~g}$ is extremely small. The current design of MEMS switch has more probability of failure in 'not-switching-on' at above $10 \mathrm{~g}$ acceleration than switchingon at below $6 \mathrm{~g}$ acceleration. Since the two failure modes are mutually exclusive, the system probability of failure is obtained by adding both failure probabilities that is 0.1648 . It is noted that this probability of failure is higher than that of MCS, but within the range of confidence intervals.

\subsection{First-Order Reliability Method (FORM)}

FORM was developed for reliability analysis in structural engineering nearly four decades back by Hasofer and Lind in 1974. Recently, FORM has been used in water resources engineering (Skaggs \& Barry, 1997; Jang, Sitar \& Kiureghian, 1994) and nuclear engineering (Cizelj, Mavko \& Oppermann, 1994). In FOSM, the performance function is linearized about the mean value, which is the reason that method is not accurate and optimal for reliability analysis. The steady state deflection $(d)$ is a function of five random variables $\left(x_{1}, x_{2}, \ldots, x_{5}\right)$ and 
MEMS switch fails if it is less than the initial gap for an acceleration of $10 \mathrm{~g}$ or more than initial gap for $6 \mathrm{~g}$, where the gap is also a random variable $\left(x_{6}\right)$. Let the performance function be defined as $G(\mathbf{x})$, which is given by Eq. (6) and the probability of failure by $P_{F}$, which is given by Eqs. (12) and (13).

The objective of FORM is to calculate an estimate of the integral of the joint PDF of input random variable $\mathbf{x}$ defined in Eq. (12). The difference between FORM and FOSM is the way to compute the reliability index $\beta$. In FORM, the reliability index is defined as the minimum distance between the failure surface and the mean surface of $\mathrm{n}$ random variables in $\mathrm{n}$-dimensional space, which is calculated by finding the 'most probable point' (MPP). In FORM, the performance function is linearized with respect to the MPP that is in turn more accurate than linearizing about the mean value. FORM follows an approach of transforming all the random variables into uncorrelated standard normal random variables and finding MPP by minimizing distance between the failure surface and origin, which is the reliability index $\beta$. The failure probability is then obtained by taking the standard normal cumulative density function of negative $\beta$.

Numerically, the MPP is determined using Hasofer-Lind and Rackwitz-Fiessler (HL-RF) algorithm (Hasofer \& Lind, 1974; Hasofer \& Lind, 1974; Rackwitz \& Fiessler, 1978). The HL-RF algorithm minimizes the distance from the origin to the failure surface with failure state equation as the equality constraint:

$$
\begin{gathered}
\text { Minimize }\|\mathbf{u}\| \\
\text { subject to } G(\mathbf{u})=0
\end{gathered}
$$

where, $\mathbf{u}$ is the vector of input random variables in standard normal space; it can be obtained by transforming input random variables $\mathbf{u}=T(\mathbf{x})$. The optimal solution $\mathbf{u}^{*}$ can be used to find the reliability index, $\beta=\| \mathbf{u}^{*}||$, which in turn yields the probability of failure $P_{F}$. It took four iterations for HL-RF algorithm to find the optimum value of $\beta$, which is ' 0.991 ' and ' $7.015^{\prime}$ ' for $10 \mathrm{~g}$ and $6 \mathrm{~g}$, respectively. Therefore, the probability of failure of MEMS Switch is 0.1606 . The value obtained lies between the confidence interval $[0.112,0.172]$ defined in Monte Carlo Simulations. The solution obtained from FORM is an approximate, unless the performance function is linear. FORM is much more computationally efficient than MCS as it requires small number of iterations to converge. However, the accuracy and efficiency of FORM is low when there are large number of random variables involved. For example, Jang et al. (1994) found that when the number of random variables are more than 100, MCS was more efficient than FORM.

\subsection{Reliability Analysis Using Surrogate Modeling}

When a performance model is computationally expensive, a surrogate model is often used to approximate the performance model using an explicit functional relationship between inputs and output. The purpose of this section is to construct a Kriging surrogate model using ooDACE MATLAB toolbox (Couckuyt, Dhaene \& Demeester, 2014) based on the values obtained from the performance model at sample points. Once the surrogate model is available, the reliability can be calculated using MCS with the surrogate model. This technique has been successfully applied previously to many performance models (Kim, Hwang \& Kwon, 2011; You, Yang, Wang \& Jia, 2009). As mentioned before, this is the most accurate reliability analysis method in this paper as it exactly models the performance at sample points and interpolates the data using Gaussian process. The accuracy of the surrogate is checked using "Root Mean Square Error" and after getting the desired accuracy, the probability of failure or reliability of MEMS switch is calculated using MCS.

\subsubsection{Latin Hypercube Sampling}

In order to build a surrogate, it is necessary to generate a set of samples in the domain of input variables and evaluate the performance functions at samples. Methods of choosing sample points are referred to as Designs of Experiments (DOE) (Kleijnen, 2005). Some examples of classic DOEs include full factorial design and central composite design. The drawback of classic DOEs is that they only sample points at the corner, faces or center of the n-dimensional cube and do not fill up the space, thus producing uncertainty in the global behavior of surrogate. These DOE's are better with less number of sample points and factors. Modern DOEs include Latin Hypercube Sampling (LHS), D-optimal design and other space-filling designs. These designs can handle a large number of factors and sample points, thus having low uncertainty. More information on different experimental designs can be found in Simpson et al. 2001.

LHS was first introduced by Mckay et al. (1979) where the range of each variable is divided into as many intervals as with the number of sampling points. The principle of LHS is that each variable will be sampled at each interval, thus producing a space filled design. LHS is used in this paper because it is computationally cheap and fast and works well with several input variables (Sacks, Welch, Mitchell \& Wynn, 1989). 200 samples are used in the range of $\left[\mu_{i}-3 \sigma_{i}, \mu_{i}+3 \sigma_{i}\right]$ for each input random variables. 


\subsubsection{Krigging Surrogate Model}

The purpose of surrogate models is to approximate performance models as closely as possible and act as an emulator while being cost and time efficient at the same time. The performance model created in Section 2 calculates the steady-state deflection of MEMS switch when input variables with uncertainty are given, but it is computationally expensive and time-consuming to perform experiments for million samples which are required for reliability analysis. This problem justifies the need of surrogate models as they can be solved the problem in seconds for million samples. There are many types of surrogate models available, such as polynomial response surface, radial basis neural networks, inductive learning and Kriging (Simpson et al. 2001). In this paper, the Kriging surrogate model is used as it is the most popular method and is prevalent in computer design of experiments. Kriging was developed by the South African mining engineer D.G. Krige for geostatics and later formalized by French engineer Georges Matheron. It expanded to spatial analysis and computer experiments when the computers became fast, and it was inexpensive to build a Kriging surrogate model. It is basically an interpolation method which predicts unknown value of a random function at a point by computing a weighted average of the known values in the neighborhood of the point. Unlike linear regression, Kriging does not depend on the shape of the function but on the form of correlation between the values of the function at nearby points. The most common type of Kriging model is Simple Kriging which is used in this paper. The basic Kriging model is given by:

$$
G(\mathbf{x})=f(\mathbf{x})+Z(\mathbf{x})
$$

where $f(\mathbf{x})$ is a trend function and $Z(\mathbf{x})$ is a Gaussian process with mean 0 and variance $\sigma^{2}$ and non-zero covariance. Simple Kriging assumes the trend function to be a known constant, i.e., $f(x)=$ constant.

\subsection{3 ooDACE Toolbox and Surrogate Construction}

The ooDACE toolbox stands for object-oriented design and analysis of computer experiments and is a MATLAB toolbox for building a Kriging surrogate model for a given dataset (Couckuyt et al. 2014). The implementation of this toolbox is very straightforward as it accepts the known function values at sample points and generates a surrogate model. LHS is used to sample 200 points with corner points. The steady state deflection is calculated at the sampled points using the performance model and ooDACE toolbox generates a Kriging fit using samples and function value at these samples. The accuracy of the surrogate model is important as it gives a measure of uncertainty in output. RMSE of 1,000 samples is used to check the accuracy, which came out to be in the order of 10-4 $\mathrm{m}$, which is sufficient to show that the surrogate is accurate within the tolerance limit and it can act as an emulator for the performance model.

Reliability analysis of MEMS switch is conducted using MCS on this surrogate with 1,000,000 samples by calculating the steady-state deflection of these samples and comparing it with the normally distributed initial gap. The input variables are normally distributed with mean and standard distribution given in Table 6. 167,952 samples fail out of 1,000,000 samples which gives us the P_F as 0.1679 . The result is justified by comparing it with the results obtained from MCS in section 3.2 where the confidence interval obtained was [0.112, 0.172], showing that it lies within the interval.

Table 8 compares the probability of failure of MEMS switch using the four different methods. All methods provide an acceptable level of accuracy except for MCS, which needs more samples to have an acceptable confidence interval.

Since most computational time is spent on calculating the steady-state response of performance function, the computational efficiency can be compared by using the number of performance evaluations. Table 8 also summarizes the total number of performance evaluations required for different methods. It is obvious that FOSM is the most computationally efficient, while the accuracy is still comparable with other methods. In addition, the sensitivity information in FOSM can provide an important information in design modification, as discussed in the following section.

Table 8. Failure probabilities from different reliability analysis methods (current design)

\begin{tabular}{ccc}
\hline Reliability Analysis Method & $\begin{array}{c}\text { Probability of Failure } \\
\text { (Current Design) }\end{array}$ & No. of performance evaluations \\
\hline Monte Carlo Simulation & {$[0.112,0.172]$} & 500 \\
FOSM & 0.1648 & 6 \\
FORM & 0.1606 & 24 \\
\hline
\end{tabular}




\section{Improving Failure Probability}

The purpose of this section is to modify the design of the MEMS switch in order to improve the current level of reliability. The failure probabilities obtained in Table 8 give an overview of the reliability of MEMS switch obtained through different methods, which is relatively large considering the importance of its use in different sectors mentioned in Introduction. Thus, there is a need to improve the reliability by changing design variables. The variable uncertain parameters in MEMS switch are the three supporting beam parameters, two material parameters and the gap between moving mass and the stationary electrode. The sensitivity of input variables with respect to steady-state deflection is given in Table 7, which shows that length and height of the supporting beam are more sensitive as compared to other variables. Therefore, a change in them is a good direction to achieve a higher reliability. But the small value of height makes it difficult to control dimensions. Therefore, a change in beam length is a good idea to increase the reliability. From the observation that most failure probability is from too small deflection at $10 \mathrm{~g}$ acceleration, design needs to be modified to make the beam less stiff, which is also supported from the positive sensitivity of the beam length. An increase in beam length will yield an increase in deflection as shown by Eq. (1) as well as sensitivity results in Table 7. Thus, the beam length is gradually increased to achieve a high level of system reliability. The MEMS switch with a beam length increased by $3.5 \%$ achieved a reliability of $99.9 \%$. Table 9 shows failure probabilities of MEMS Switch with improved design obtained through different reliability methods.

Table 9. Failure probabilities of improved design

\begin{tabular}{ll}
\hline Reliability Analysis Method & Probability of Failure (Optimum Design) \\
\hline Monte Carlo Simulation & 0 (500 Samples) \\
FOSM & 0.00026 \\
FORM & 0.00023 \\
Surrogate Modeling & 0.000362 \\
\hline
\end{tabular}

\section{Conclusions and Discussions}

In this paper, 1D mass-spring-damper-contact model of MEMS acceleration switch is proposed that can be used for reliability analysis caused by geometry tolerances and material variability. A systematic model simplification and validation is performed using a high-fidelity finite element model, which is also used for model calibration. It is shown that the dynamic responses of the switch from the 1D simplified model and finite element model are in good agreement.

Four different reliability analysis methods are utilized to estimate the probability of failure. All methods yield a similar level of reliability, but the first-order second-moment method (FOSM) was most inexpensive in terms of the number of function evaluations. In addition, the sensitivity provides a useful information to improve the level of reliability. From sensitivity analysis, the length of the folded beam was increased by $3.5 \%$, which improves the reliability from $84 \%$ to $99.9 \%$.

\section{Acknowledgement}

This research has been supported by Defense Acquisition Program Administration (DAPA) and Agency for Defense Development (ADD) in Korea.

\section{References}

Agarwal, N., \& Aluru, N. R. (2009). A Domain Adaptive Stochastic Approach for Analysis of MEMS under $\begin{array}{lllll}\text { Uncertainties. Journal of } & \text { 7662-7688. }\end{array}$ https://doi.org/10.1016/j.jcp.2009.07.014

Cai, H., Ding, G., Yang, Z., Su, Z., Zhou, J., \& Wang, H. (2008). Design, Simulation and Fabrication of a Novel Contact Enhanced MEMS Inertial Switch with a movable Contact Point. Journal of Micromechanics and Microengineering, 18(11), 1-10. https://doi.org/10.1088/0960-1317/18/11/115033

Choi, K. K., \& Kim, N. H. (2004). Structural Sensitivity Analysis and Optimization 1", Springer Publication, 2022. ISBN 978-0-387-27169-9.

Cizelj, L., Mavko, B., \& Oppermann, H. R. (1994). Application of first and second order reliability methods in the 
safety assessment of cracked steam generator tubing. Nuclear Engineering and Design, 147(3), 359-368. https://doi.org/10.1016/0029-5493(94)90218-6

Couckuyt, I., Dhaene, T., \& Demeester, P. (2014). ooDACE Toolbox: A Flexible Object-Oriented Kriging Implementation. Journal of Machine Learning Research, 15, 3183-3186.

Dunnigan, K. (2008). Confidence Interval Calculation for Binomial Proportions. MWSUG Conference.

Frobenius, W. D., Zeitman, S. A., White, M. H., O'Sullivan, D. D., \& Hamel, R. G. (1972). Micro miniature Ganged Threshold Accelerometers Compatible with Integrated Circuit Technology. IEEE Transactions on Electron Devices, ED-19, 37-40. https://doi.org/10.1109/T-ED.1972.17368

Hamid, M. L., \& Parviz, E. N. (1994). Continuous Contact Force Model for Impact Analysis in Multibody Systems, Nonlinear Dynamics, 5, Kluwer Academic Publishers, 193-207.

Hasofer, A. M., \& Lind, N. C. (1974). Exact and Invariant Second-Moment Code Format. Journal of Engineering Mechanics Division ASCE, 100(EMI), 111-121.

Hsu, T. R. (2003). Mems Packaging (EMIS Processing). Institute of Engineering and Technology, 9-11. ISBN-10: 0863413358.

Jang, Y. S., Sitar, N., \& Kiureghian, A. D. (1994). Reliability analysis of contaminant transport in saturated porous media. Water Resources Research, 30(8), 2435-2448. https://doi.org/10.1029/93WR03554

Kim, J. B., Hwang, K. Y., \& Kwon, B. I. (2011). Optimization of Two-Phase In-Wheel IPMSM for Wide Speed Range by Using the Kriging Model Based on Latin Hypercube Sampling”, Magnetics, IEEE, 47(5), 1078 1081. https://doi.org/10.1109/TMAG.2010.2096409

Kim, J. M. (2004). A Study on MEMS Acceleration Switch”, Chonbuk National University.

Kleijnen, J. P. C. (2005). An overview of the design and analysis of simulation experiments for sensitivity analysis", 2005, European Journal of Operational Research, 164(2), 287-300. https://doi.org/10.2307/1268522

Lee, J. I., Eun, Y., Jung, H. I., Choi, J., \& Kim, J. (2011). A Novel Accelerometer Based on Contact Resistance of Integrated Carbon Nanotubes. MEMS, IEEE 24 International Conference, 533-536. https://doi.org/10.1109/MEMSYS.2011.5734479

Lee, J. I., Song, Y., Jung, H. K., Choi, J., Eun, Y., \& Kim, J. (2011). Carbon Nanotubes Integrated Inertial Switch for Reliable Detection of Threshold Acceleration. Solid State Sensors, Actuators and Microsystems Conference, 711-714. https://doi.org/10.1109/TRANSDUCERS.2011.5969201

Loke, Y., Mckinnon, G. H., \& Brett, M. J. (1991). Fabrication and Characterization of silicon micromachined threshold accelerometers. Sensors and Actuators, 235-240. https://doi.org/10.1016/0924-4247(91)80020-P

Massad, J. E., Sumali, H., Epp, D. S., \& Dyck, C. W. (2005). Modeling, Simulation and Testing of the Mechanical Dynamics of an RF Mems Switch. International Conference on MEMS, NANO and smart systems, IEEE, 237-240. https://doi.org/10.1109/ICMENS.2005.77

Matsunaga, T., \& Esashi, M. (2002). Acceleration Switch with Extended Holding Time using Squeeze Film Effect for Side Airbag Systems", Sensors and Actuators A: Physical, 100, 10-17. https://doi.org/10.1016/S09244247(02)00039-0

McKay, M. D., Beckman, R. J., \& Conover, W. J. (1979). A Comparison of Three Methods for Selecting Values of Input Variables in the Analysis of Output from a Computer Code. Technometrics, 21(2), 239-245.

Michaelis, S., Timme, H. J., Wycisk, M., \& Binder, J. (2000). Additive Electroplating Technology as a post CMOS process for the production of MEMS acceleration threshold switches for transportation applications. Journal of Micromechanics and Microengineering, 120-123. https://doi.org/10.1088/0960-1317/10/2/304

Rackwitz, R. (1976). Practical Probabilistic Approach to Design. Bulletin, 112, Comite European du Beton, Paris, France.

Rackwitz, R., \& Fiessler, B. (1978). Structural Reliability under Combined Random Load Sequences. Computers and Structures, 9(5), 484-494. https://doi.org/10.1016/0045-7949(78)90046-9

Sacks, J., Welch, T. J., Mitchell, W. J., \& Wynn, H. P. (1989). Design and analysis of computer experiments", Statistical Science, 4(4), 409-431. https://doi.org/10.1214/ss/1177012413

Selvakumar, A., Yazdi, N., \& Najafi, K. (2000). A wide range micro machined threshold accelerometer array and interface circuit. Journal of Micromechanics and Microengineering, 118-125. https://doi.org/10.1088/0960- 
$1317 / 11 / 2 / 306$

Simpson, T. W., Poplinski, J. D., Koch, P. N., \& Allen, J. K. (2001). Metamodels for Computer-based Engineering Design: Survey and recommendations. Engineering with Computers, 17(2), 129-150. https://doi.org/10.1007/PL00007198

Skaggs, T. H., \& Barry, D. A. (1997). The first-order reliability method of predicting cumulative mass flux in heterogeneous porous formations. Water Resources Research, 33(6), 1485-1494. https://doi.org/10.1029/97WR00660

Tonnesen, T., Ludtke, O., Noetzel, J., Binder, J., \& Mader, G. (1997). Simulation, Design and Fabrication of electroplated acceleration switches. Journal of Micromechanics and Microengineering, 237-239. https://doi.org/10.1088/0960-1317/7/3/042

You, H., Yang, M., Wang, D., \& Jia, X. (2009). Kriging Model combined with Latin Hypercube Sampling for Surrogate Modeling of Analog Interpreted Circuit Performance. Quality of Electronic Design, IEEE, $554-$ 558.

Zhou, C. C., Tu, C. L., Wong, F. X., Gong, H. W., Lian, P., He, C., \& Ye, X. S. (2014). A Low-power, Wrist-worn Device for Long Time Heart Rate Monitoring and Fall Detection", (ICOT) IEEE International Conference, 33-36.

\section{Copyrights}

Copyright for this article is retained by the author(s), with first publication rights granted to the journal.

This is an open-access article distributed under the terms and conditions of the Creative Commons Attribution license (http://creativecommons.org/licenses/by/4.0/). 